\title{
REVIEW OF OBSERVATIONAL DATA ON RR LYRAE STARS
}

\author{
G. van HERK
}

Leiden Observatory, Leiden, The Netherlands

The data on which my work on the secular parallaxes of RR Lyrae stars is based (Bull. Astron. Inst. Neth. 18) were in many respects so incomplete that $\mathrm{I}$ have tried to interest astronomers to get a more complete set of data. The number of stars for which I had a proper motion was only 168 , and for which a radial velocity was known, was 180 , with an overlap of only 138 stars. The accuracy of the proper motions was certainly unsatisfactory for $43 \%$ of the total. The greatest trouble in dealing with such insufficient numbers arises when one wants to subdivide the material into groups which are homogeneous from a physical point of view. Many subdivisions, in making up my paper, were not tried at all, simply because the material was inadequate.

In recent years plenty of work has been done by various investigators, of which I will at this point only mention the work on proper motions done at the Leander McCormick Observatory, and the great number of radial velocities determined by Dr. Clube and his associates. I do not, however, believe we are yet in a position to consider the whole subject as finished. Discussions about space motions, as given by Professor Oort in the book Stellar Structures, Volume V, will, at this time, be hardly improved upon. I still feel we should increase the number of stars substantially in order to get a better statistical discussion possible. This means we have to go to fainter stars. Plenty of these stars will be found on the plates which have served to make the Charts of the Carte du Ciel, which means we have at least one old position available for proper motions. At Leiden we are now engaged in the determination or redetermination of the proper motions of 430 R R Lyrae stars.

Another point to worry about can be best seen in Figure 1, where the stars with known proper motions are plotted in a coordinate system showing the equatorial poles, the equator, and the $45 \mathrm{deg}$ declination circles. The subdivision according to periods, which was followed mainly in the Leiden investigation, shows how meager the data are from the southern hemisphere. It is very much hoped this will be remedied, were it only to serve as a valuable check on the systematic results from the material of the northern sky. It would require an independent determination of the secular parallaxes of reference stars as was done by Binnendijk and others.

From several authorities on image-converters, I learned that medium-sized telescopes (of which there exist plenty) equipped with image converters could help very well in solving the deficiency in our knowledge with respect to radial velocities of RR Lyrae stars. These velocities are usually so large that a lesser accuracy of one determination as the classical methods yield, would not be too harmful. I would like to mention, incidentally, that we should observe many radial velocities, if only to complete the extensive search by Dr Plaut for RR Lyrae stars.

A very satisfactory improvement can be reported on the photometric data. I do not 

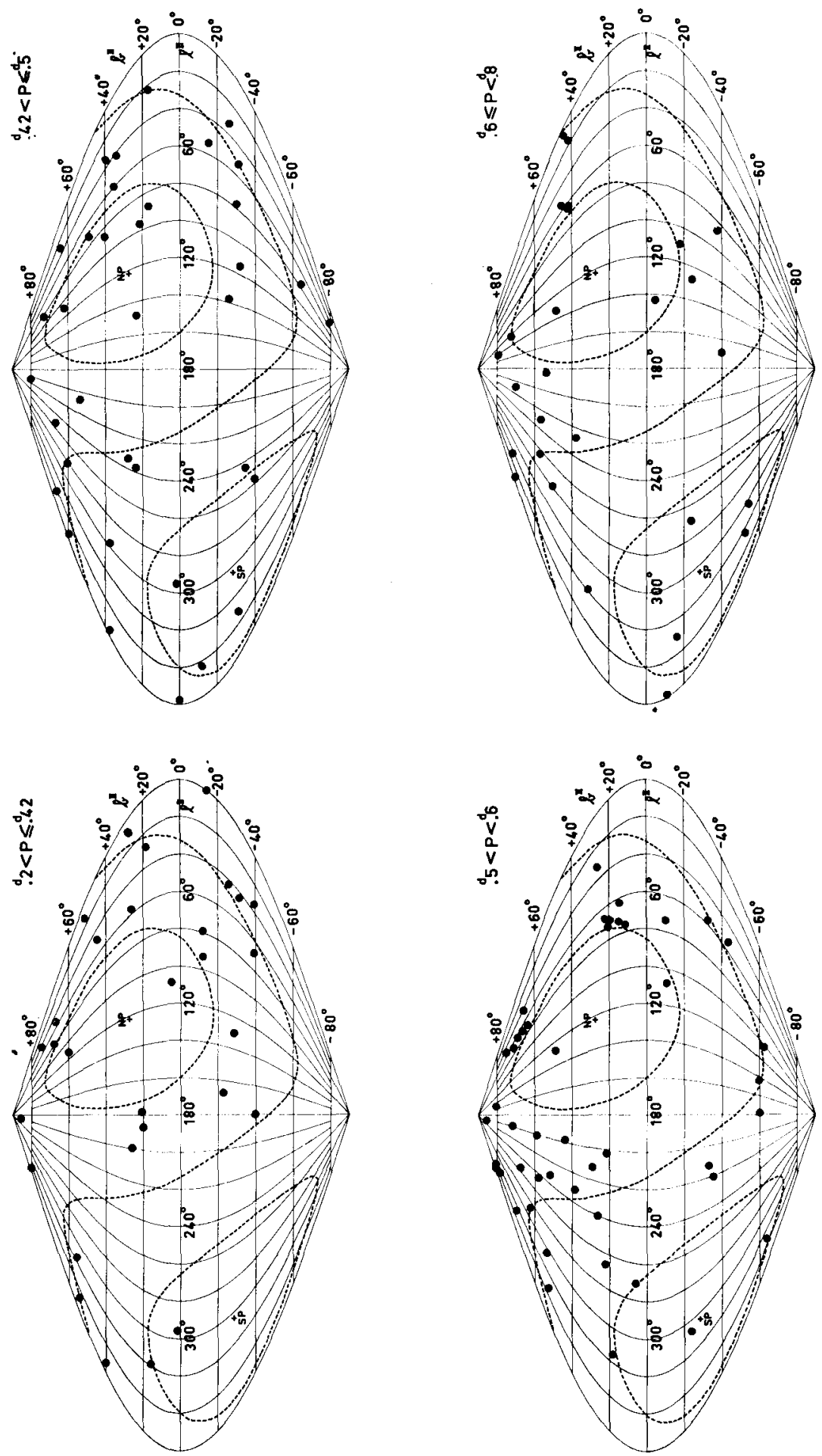

$\stackrel{\dot{m}}{\dot{m}}$ 
know exactly how much has been achieved lately by the group of Drs Fitch and Johnson, but, together with the earlier work done by Dr Kron, I believe we have now good photometric data for some 200 stars. If we want to increase our knowledge by going to fainter stars, it is to be hoped that this list will be extended as well, as I remember with great distress how poor most of these data have been in the good old days.

\section{TABLE I}

\begin{tabular}{|c|c|c|c|c|c|}
\hline & \multicolumn{4}{|c|}{$\Delta s$ for RR Lyrae stars } & \multirow[b]{2}{*}{ Alania } \\
\hline & Preston & Kinman & Cape & Clube & \\
\hline SW And & 0 & & & & 0 \\
\hline SW Aqr & 5 & & & 11 & \\
\hline SX Aqr & 9 & & & 9 & \\
\hline X Ari & 10 & & & & 11 \\
\hline RS Boo & 2 & & & & 4 \\
\hline RZ Cep & 5 & & & & 5 \\
\hline RU Cet & 9 & & & 7 & \\
\hline RV Cet & & & 3 & 9 & \\
\hline RX Cet & & & 2 & 5 & \\
\hline UU Cet & 4 & & & 4 & \\
\hline S Com & 7 & & & & 8 \\
\hline W Crt & 3 & & & 0 & \\
\hline SU Dra & 10 & & & & 10 \\
\hline SW Dra & 3 & & & & 4 \\
\hline XZ Dra & 3 & & & & 5 \\
\hline RX Eri & 9 & 6 & & & \\
\hline SV Eri & 9 & & & 5 & \\
\hline SS For & & & 13 & 2 & \\
\hline $\mathrm{V}$ Ind & & & 10 & 7 & \\
\hline RR Leo & 8 & & & & 4 \\
\hline U Lep & 9 & & & 7 & \\
\hline TV Lib & 2 & & & 3 & \\
\hline RZ Lyr & 9 & & & & 5 \\
\hline V 445 Oph & 1 & & & 3 & \\
\hline AV Peg & 0 & & & & 1 \\
\hline BH Peg & 5 & & & & 8 \\
\hline V $440 \mathrm{Sgr}$ & 5 & & & 9 & \\
\hline RU Scl & & & 9 & 6 & \\
\hline RV UMa & 8 & & & & 6 \\
\hline
\end{tabular}

Finally, we had at our disposition the metal index as defined by Preston. In 1965, $\Delta S$ was known for some 100 stars, which meant again that no extensive use could be made of $\Delta S$ in subdividing the material. Now, the determination of $\Delta S$ has to be done near minimum light according to the originator of this index, which means it is difficult to determine, and perhaps well-nigh impossible for fainter stars. It is to this end that I have asked permission, since 1965 , to have the Leiden observers, working at the Leiden Southern Station, observe RR Lyrae stars in the 5-colour system of $\mathrm{Dr}$ Walraven. Figure 2 shows the colours picked out by Walraven in comparison with the 


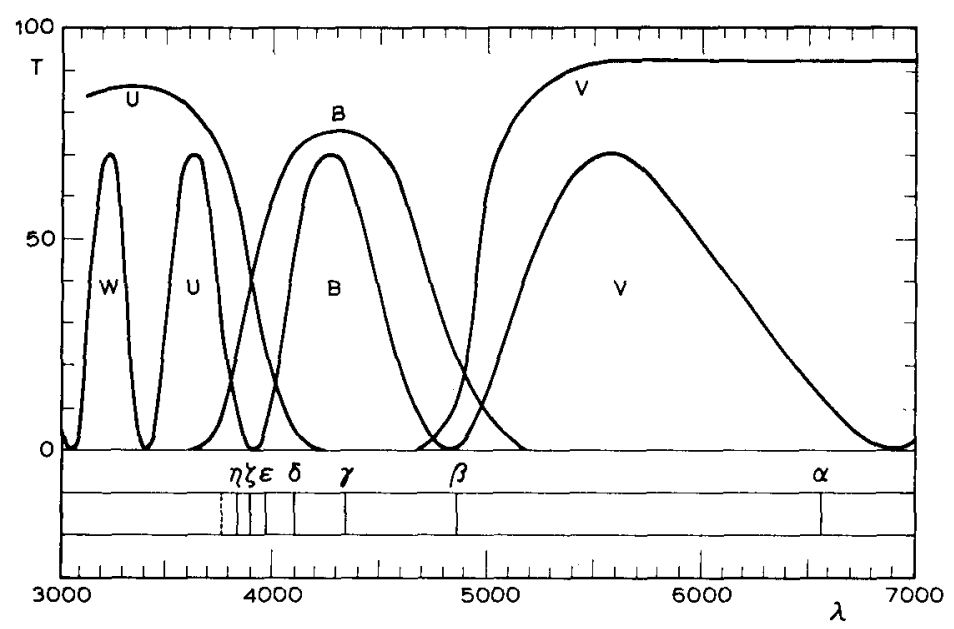

Fig. 2. Transmission curves of spectro-photometer. For comparison the Transmission curves as used in the U, B, V system are also shown.

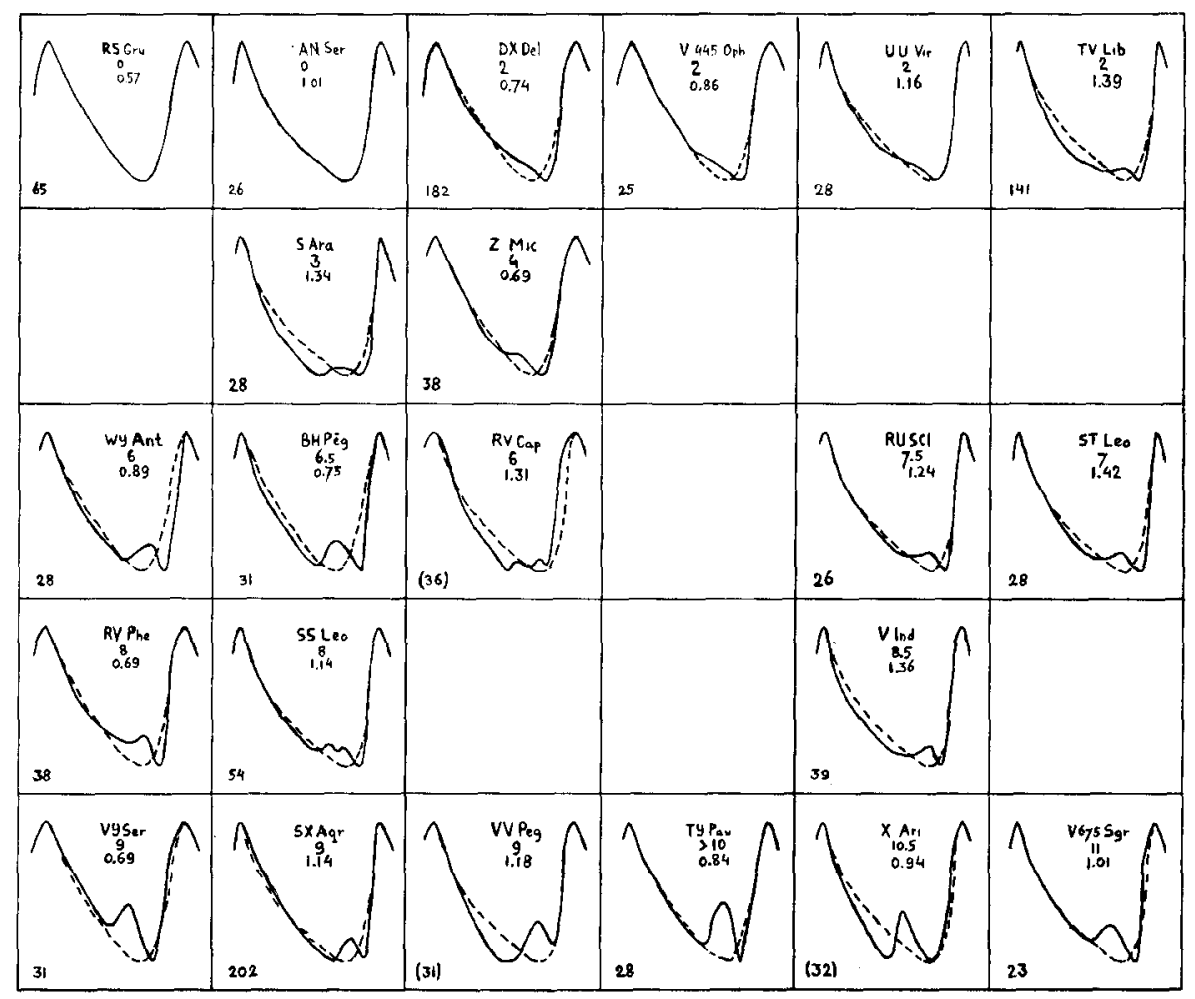

Fig. 3. 
international UBV system. It is to be noted that Walraven's $U$ is almost free from hydrogen lines, and covers a region where metallic lines are often abundant.

Unfortunately, I can show only results for some 20 stars. We are now engaged in making great efforts to observe some 80 stars more, including as many $c$-type stars as we can get. In what I want to show to you, only Bailey a-type stars are involved. Figure 3 presents normalized lightcurves for 22 stars, in Walraven's U-colour, and with known $\Delta S$. These lightcurves are fully drawn, and are given in five lines with increasing $\Delta S$. On each line the amplitudes have been used as a second subdivision. This small sample of stars has, so far, convinced me that the so-called secondary bump increases in magnitude with increasing $\Delta S$, or with decreasing metal index. The dotted lines are the two curves found for the two stars with $\Delta S=0$, given in the left-hand top corner. Which one was reproduced depended on the amplitude, but I wished I had at my disposal at least 5 stars with $\Delta S=0$ to match better the different amplitudes. I want to note here that the phase of the maximum of the bump seems to be almost
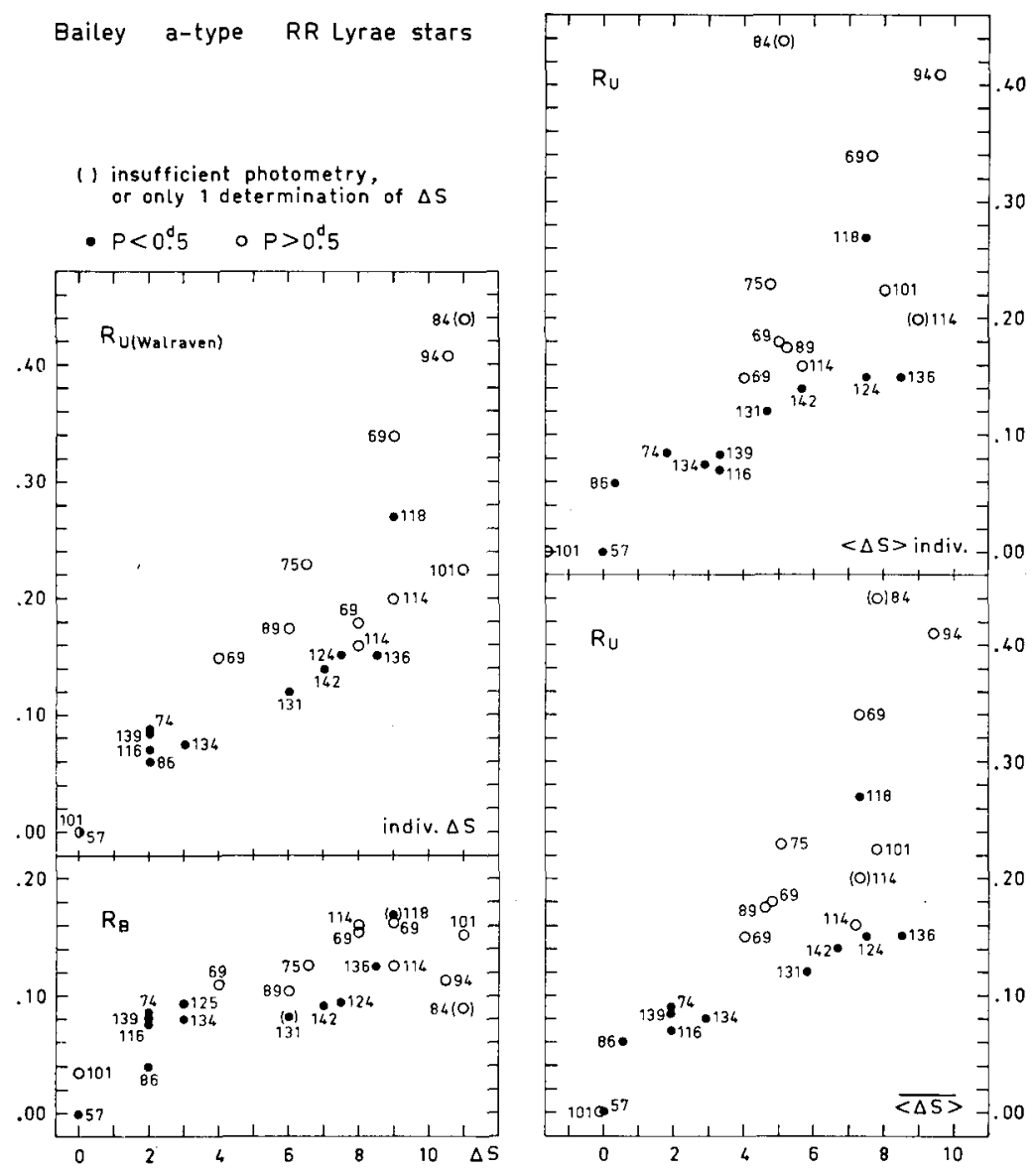

Fig. 4. 
constant. From two ways of determining this phase, we found an average value of 0.685 , with a standard deviation for this average of 0.015 on the assumption that all bump phases are the same.

Returning to the height of the bump, we tried to write down a figure for it. As we do not know the base level from which to measure the height of the bump, we used an 'undisturbed' lightcurve and measured the greatest deviation from the actual curve to the undisturbed one at the place where the bump occurs. We expressed this greatest deviation in the amplitude as the unit. We hope this quantity is practically independent of all uncertainties of the effect of reddening, as only one colour is involved. For stars with heavy bumps, this procedure to draw a smooth, undisturbed lightcurve can become rather arbitrary, and here we used the relative height of the secondary maximum above the minimum with respect to the total amplitude. These ratios were plotted against $\Delta S$ as given in the left-hand side of Figure 4. It seems quite likely there exists a continuous change of this ratio with $\Delta S$. The same ratio has been determined for Walraven's B-colour, which you know is observed at the same time as the other colours. The slope we notice in the first diagram is now far less pronounced as can be seen from the graph in the left-hand bottom corner. The scatter one notices is partly due to the uncertainty in $\Delta S$, which was estimated by Preston as 1 to 2 units. We have tried to get some insight in the $\Delta S$ accuracy by studying all the original data given by Preston and by Kinman. The $\Delta S$ on the right-hand side of Figure 4 are the slightly revised values that we found.

In Figure 5 for a few of the stars the individual $\Delta S$ have been plotted against phase. We did it for all stars, and we could not convince ourselves that it is strictly necessary

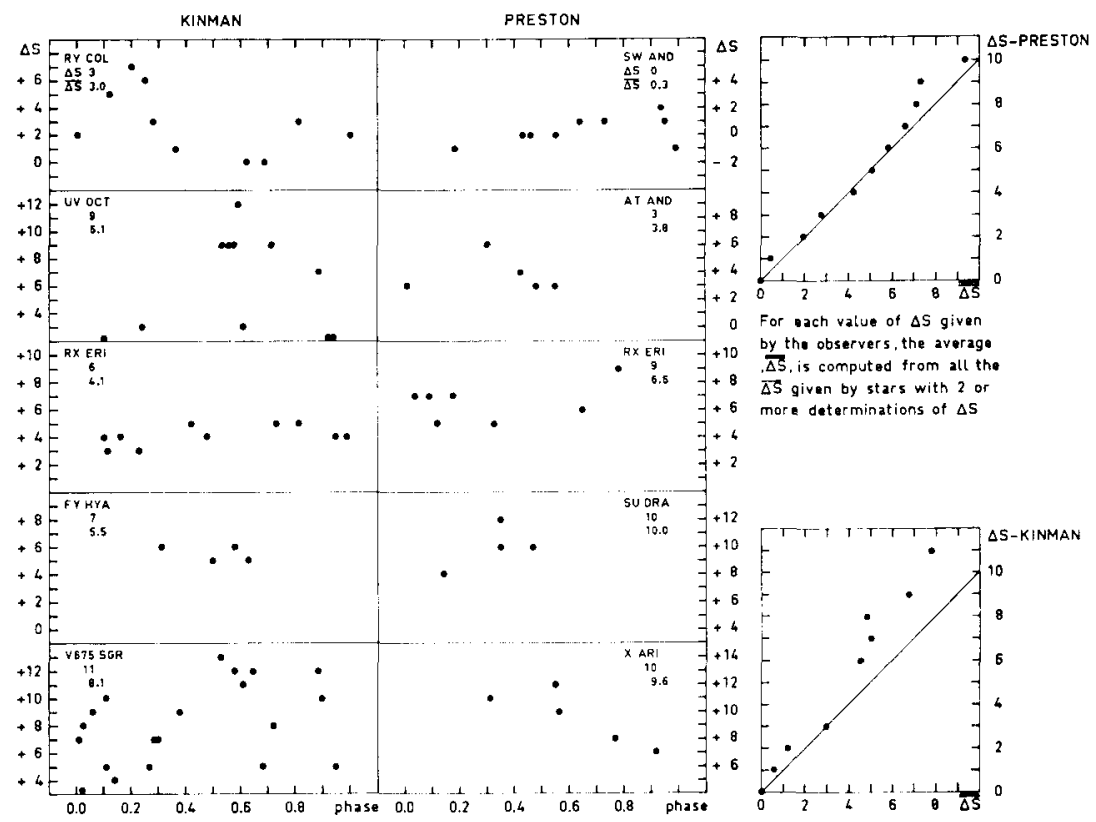

Fig. 5. 
to have the $\Delta S$ determined near minimum light. Our sample shows that it is easy to doubt any real change of $\Delta S$ with phase. We have assumed for a moment that all determinations should have given the same answer, and therefore we have taken the average of all values observed for each star. The $\Delta S$ thus found have been used in Figure 4 in the top right-hand corner. If we now could believe that the averages just mentioned are better values for the $\Delta S$ assigned to the star, we can average the new values per group of each of the 12 values given for $\Delta S$ by the authors, namely from 0 to 11. A plot in the right hand side of Figure 5 shows the deviations of these newly computed $\Delta S$ against the original values. For Preston there is hardly a difference of importance; only at $\Delta S=9$ we see some discrepancy. This is not true for Kinman's values. Here some pronounced systematic effect turns up for the higher values. A look at the scatter of Kinman's individual values makes me still hesitate to believe that we have to accept anything else but the ordinary scatter from observational results. There is one question I found unanswered: Why have never negative values for $\Delta S$ been introduced? Both Preston and Clube have found negative values, but have never used them. Kinman has observed several stars for which all individual determinations were estimated to be zero, with not a single deviation. These seem to contradict the estimated error of 1-2 units in $\Delta S$.

The overall averages for the $\Delta S$ have been used in the right-hand bottom part of Figure 4. In essence, the scatter in all three diagrams has not changed very much. We could have minimized the scatter by drawing freehand undisturbed lightcurves for the cases with large $\Delta S$, but we have preferred to wait till we learn from where we have to reckon these disturbances in the actual lightcurves. Whatever the explanation of the relation between $\Delta S$ and the height of the bumps, I am inclined to believe that we could use this type of observation to determine the metal index for stars which are too faint to be coped with in the classical way.

\section{DISCUSSION}

Christy: The bumps you found in the $U$ light curves of RR Lyrae variables and their correlation with $Z$ is very interesting. In my model calculations I sometimes found bumps in this region and sometimes did not, so I had concluded that their appearance (perhaps due to internal interference) was very sensitive to some model parameters. Perhaps, since I believe the luminosity and metal content are inversely correlated, one can understand the dependence of bump intensity on metal content as the indirect influence of the luminosity and the sensitivity to model parameters that I found. Further work will be necessary to understand this very significant correlation.

Warren: I have done UBV photometry on a number of RR Lyrae stars and haven't found any bumps in the light curves.

Van Herk: The light curves I showed were in the intermediate-band Walraven U, not the broadband Johnson $\mathbf{U}$. 